\title{
Small Bowel Obstruction Caused By Retained Placenta Post Emergency Cesarean Section
}

\author{
Sean Park ${ }^{1,2}$, Cino Bendinelli ${ }^{1}$, John Bailey ${ }^{2}$ \\ Department of ${ }^{1}$ General Surgery and ${ }^{2}$ Obstetrics and Gynecology, John Hunter Hospital, University of Newcastle, Newcastle, NSW. \\ Australia.
}

\section{Corresponding Author:}

Dr. Sean (Sang Woo) Park

Email: seannypark@hotmail.com

This is an Open Access article distributed under the terms of the Creative Commons Attribution License (creativecommons.org/ licenses/by/3.0).

Received

Accepted

February 8, 2017

Published

April 15, 2017

May 15, 2017

\begin{abstract}
Background: This case report illustrates a rare and unusual presentation of small bowel obstruction caused by a retained placenta following cesarean section. Case Report: A 38 year old G3P2 multiparous female patient was admitted to hospital with spontaneous rupture of membranes and premature labour on the background of previous successful cesarean and Ventouse deliveries. Following failed attempts at vaginal delivery, the patient underwent an emergency cesarean section. On day one post-operation, the patient developed significant central abdominal pain out of proportion to the surgery, with associated bilious vomiting. A diagnosis of small bowel obstruction was confirmed on $\mathrm{X}$-ray and CT-abdomen and a trial of conservative management failed. A laparotomy was subsequently performed which revealed a retained placenta superior to the uterus causing small bowel obstruction. The retained placenta was surgically removed, which resulted in the resolution of the bowel obstruction. Conclusion: We report an uncommon but important cause of small bowel obstruction secondary to cesarean section retained placenta. Although this is a rare presentation, it is an important differential diagnosis for the surgeon to keep in mind in the post-operative period.
\end{abstract}

Keywords: Abdominal Pain, Cesarean Section, Placenta, Pregnancy, Rupture.

\section{Introduction}

The mode of cesarean delivery has become increasingly common in the Western society over the past few decades, accounting for up to $30 \%$ of all births. In comparison, only $4.5 \%$ of deliveries were performed by cesarean delivery in 1965 [1]. The dramatic rise in cesarean delivery is attributed to various factors like risk of uterine rupture, patient's preference and increasing maternal age $[2,3]$. Although cesarean delivery has improved maternal and post-natal outcomes, especially for challenging pregnancies and is deemed generally safe in modern obstetrics, it does not come without a cost. It carries a risk for potential intra-operative and post-operative morbidities such as adhesions, bowel injury, ureteral injury and increased risk of pathological placenta in subsequent pregnancies.
We describe an unusual presentation of acute small bowel obstruction caused by an intra-abdominally retained placenta following emergency cesarean section. It is a rare surgical complication of cesarean section, likely due to a technically difficult access to the uterus as a result of significant intraabdominal adhesions and subsequent migration of placenta tissue.

\section{Case Report}

A 38-year-old G3P2 multiparous female presented to hospital with spontaneous rupture of membranes and premature labor. Her two previous pregnancies were successfully carried to term and delivered by cesarean section for the first pregnancy due to macrosomia and Ventouse for the second. The patient's other significant past medical and surgical 
history included iron deficiency anemia, obesity, and a left salpingectomy. Due to a pathological cardio-tocography, an instrumental delivery in theatre was attempted. A Ventouse was applied and after two pulls with contractions, no fetal descent was apparent. Consequently, an emergency cesarean section was carried out which entailed extensive adhesiolysis between the uterus, bladder and omentum and a difficult access through a small window in the upper uterus. A healthy baby was delivered, and the uterine cavity was confirmed empty with complete delivery of the placenta. The total blood volume loss was $750 \mathrm{ml}$ and there was no intra-operative complication.

On day one post-cesarean section, the patient had a rapid response call for central abdominal pain and associated nausea and bilious vomiting. An abdominal X-ray demonstrated dilated small bowel loops with possible small bowel obstruction or ileus. She was conservatively managed with a nasogastric tube on suction which immediately drained approximately $500 \mathrm{ml}$ on insertion. She remained hemodynamically stable without frank evidence of complications from small bowel obstruction. Blood test demonstrated lactate $1.4 \mathrm{mmol} / \mathrm{L}$ (normal: $0.5-1$ ), white cell count $17.3 \times 10^{9} / \mathrm{L}\left(4.5-11 \times 10^{9} / \mathrm{L}\right)$, hemoglobin $107 \mathrm{~g} / \mathrm{L}$ (138-172), neutrophils $16.1 \times 10^{9} / \mathrm{L} \quad\left(2-7 \times 10^{9}\right)$, platelets $154 \times 10^{9} / \mathrm{L}\left(150-400 \times 10^{9}\right)$. However, the patient complained of progressively worsening abdominal pain, and a computed tomography scan of the abdomen on day two [Fig.1] confirmed small bowel obstruction with a transition point in midabdomen superior to the uterus. However, a clear visualisation of the cause was difficult as a result of concurrent low-density free fluid as demonstrated in Figure 1.

On day three, the obstruction did not resolve and the non-operative management was abandoned. At laparotomy fragments of retained placenta were found intra-peritoneally in the right iliac fossa. These were causing acute inflammation,

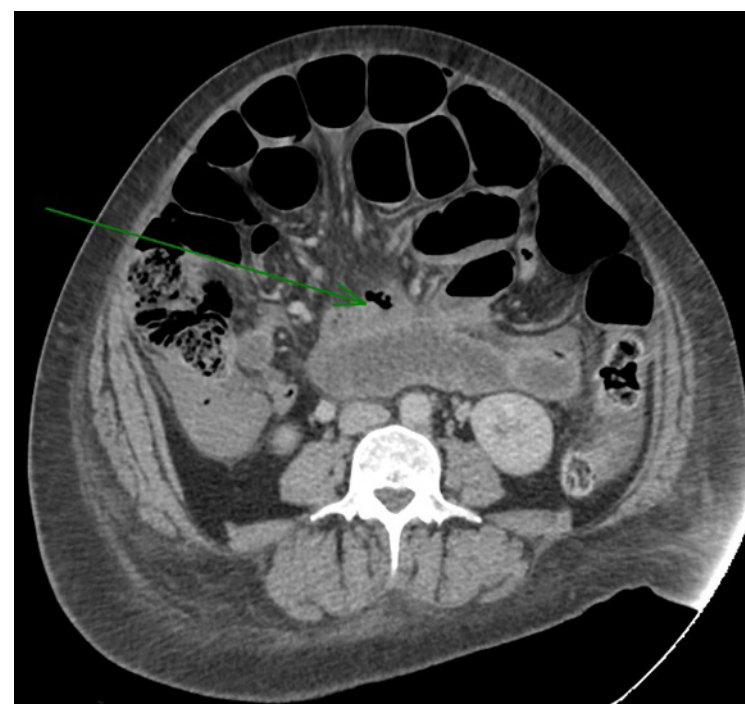

Fig.1: Computed tomography demonstrating small bowel obstruction with a transition point (green arrow) superior to the uterus.

adhesions and obstruction of distal small bowel. Some other parts of the placenta were found to be firmly adhering to the caecum. The main retained fragments of placenta [Fig.2] were easily removed. The placenta implanted on the cecum had to be peeled off gently but using some force. The rest of the abdominal cavity was normal at intraoperative examination. The histopathology confirmed a placental tissue $(90 \times 65 \times 30 \mathrm{~mm}, 102.2$ grams $)$ with no umbilical cord. The patient made a slow, but uneventful recovery and was discharged safely. The $\beta$-HCG hormone levels were not followed biochemically due to her progressive clinical improvement. The subsequent out-patient followup in four weeks revealed continual improvement.

\section{Discussion}

To the best of our knowledge this is the first report of intra-abdominally retained placenta following cesarean section resulting in an acute small bowel obstruction. Complications of cesarean delivery can result from diverse factors including maternal and fetal health factors, surgical technique, and the timing and nature of operation [2]. Intraabdominally retained placenta in this case was 
largely attributed to the emergent nature of the surgery, the presence of significant adhesions secondary to previous cesarean and pelvic surgery, high body mass index and consequent challenging surgical access. This is supported by the current literature that cesarean section is associated with various potential peri-operative complications, including adhesions, infections and wound complications, bleeding, bowel injury and obstruction, prolonged post-operative recovery, and delays in delivery.

Abdominal and pelvic adhesions are fibrous, band-like structures that form between abdominal organs or between the peritoneum and abdominal wall when trauma such as infection and surgery induces inflammation and disrupts normal tissue $[4,5]$. Its formation entails a distinct and complex inflammatory cascade with simultaneous epithelisation of the peritoneum over the whole surface immediately after surgery, unlike skin and other tissue that heals from the edges of disrupted epithelium $[4,6,7]$. It is reported that the incidence of adhesion formation ranges from $46-65 \%$ after a single cesarean intervention [3], and gynaecological and colorectal surgery have been demonstrated to be associated with higher incidence of adhesion development [4]. At the same time, the rate of intestinal obstruction after cesarean section is quoted in the literature to be 0.5 per 1000 and $1 \%$ after single and multiple cesarean sections, respectively [8].

The presumed mechanism in this case is a failure of complete removal of the placenta through the small superior uterine window. The placenta likely migrated out of the uterine cavity causing an inflammatory reaction, adhesions and small bowel obstruction. Although ectopic placental tissue may have been formed on the small bowel from a heterotopic pregnancy, it is unlikely for an ectopic tissue of term placenta to have an independent ability to infiltrate viscera outside the uterus in the absence of decidua basalis

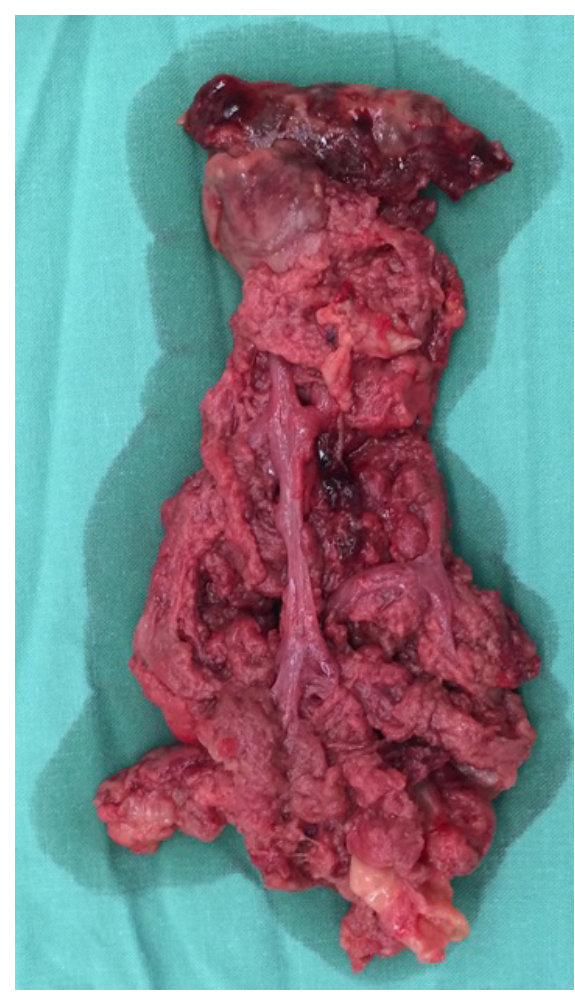

Fig.2: Retained fragments of placenta.

or stimulus for the implantation, being responsible for the obstruction. The retained placenta tissue outside the uterus can however certainly induce cytokines that would potentiate chemotactic and inflammatory cascades, leading to an adhesive state of the viscera. The inflammatory process could be medically suppressed as a temporary measure, but the offending placenta needs to be surgically removed to relieve the obstruction and prevent further complications.

Although it can be difficult to demonstrate a direct causal relationship between adhesions and post-operative morbidity and currently there is no identified predictive factor for adhesion formation [9], a number of associated risk factors have been implicated: infection, hypoxic injury, tissue desiccation, poor handling of tissue, presence of intra-peritoneal blood and foreign bodies $[6,7,9]$. The presence of adhesion makes the surgical field hostile and can potentially increase the risk of perioperative complications. Therefore, the surgical 
techniques that involve careful handling of tissues, the avoidance of free blood, and the maintenance of tissue moisture are warranted [6,7], and further studies are required to evaluate effective ways to minimise the adhesion development. Moreover, careful consideration and reduction of unnecessary primary and repeat cesarean interventions whenever possible may be the simplest way to minimise and avoid the multiple potential maternal and fetal complications.

\section{Conclusion}

A retained placenta causing small bowel obstruction following cesarean section is an unusual and unexpected clinical scenario. However, it is important for clinicians to be aware of this entity as a rare potential cause of post-operative abdominal pain and small bowel obstruction in women undergoing cesarean section.

Contributors: SP: manuscript drafting, literature review; CB: manuscript revision, literature review. JB: manuscript revision. SP will act as guarantor. All authors approved the final version of the manuscript.

Funding: None; Competing interests: None stated.

\section{References}

1. Taffel SM, Placek PJ, Liss T. Trends in the United States cesarean section rate and reasons for the 1980-85 rise.
Am J Public Health. 1987;77:955-959.

2. Lyell DJ. Adhesions and perioperative complications of repeat cesarean delivery. Am J Obstet Gynecol. 2011;205(6 Suppl):S11-8.

3. Silver RM, Landon MB, Rouse DJ, Leveno KJ, Spong CY, Thom EA, et al. Maternal morbidity associated with multiple repeat cesarean deliveries. Obstet Gynecol. 2006;107:1226-1232.

4. Darmas B. Use of barrier products in the prevention of adhesion formation following surgery. J Wound Care. 2008; 17:405-408.

5. Diamond MP, Freeman ML. Clinical implications of postsurgical adhesions. Hum Reprod Update. 2001;7:567-576.

6. Practice Committee of American Society for Reproductive Medicine in collaboration with Society of Reproductive Surgeons. Pathogenesis, consequences, and control of peritoneal adhesions in gynecologic surgery. Fertil Steril. 2008;90(5 Suppl):S144-149.

7. Liakakos T, Thomakos N, Fine PM, Dervenis C, Young RL. Peritoneal adhesions: etiology, pathophysiology, and clinical significance. Recent advances in prevention and management. Dig Surg. 2001;18:260-273.

8. Andolf E, Thorsell M, Kallen K. Cesarean delivery and risk for postoperative adhesions and intestinal obstruction: a nested case-control study of the Swedish Medical Birth Registry. Am J Obstet Gynecol. 2010;203:406.e1-6.

9. Alpay Z, Saed GM, Diamond MP. Postoperative adhesions: from formation to prevention. Semin Reprod Med. 2008;26:313-321. 